\title{
Extracts and compounds with anti-diabetic complications and anti-cancer activity from Castanea mollissina Blume (Chinese chestnut)
}

\author{
Lin Zhang ${ }^{1}$, Hui-yuan Gao ${ }^{2 *}$, Masaki Baba ${ }^{3}$, Yoshihito Okada ${ }^{3}$, Toru Okuyama ${ }^{3}$, Li-jun Wu ${ }^{2}$ and Li-bin Zhan ${ }^{4,1^{*}}$
}

\begin{abstract}
Background: Castanea mollissima Blume (Chinese chestnut), as a food product is known for its various nutrients and functional values to the human health. The present study was carried out to analyze the anti-diabetic complications and anti-cancer activities of the bioactive compounds present in C. mollissima.

Methods: The kernels (CK), shells (CS) and involucres (CI) parts of C. Blume were extracted with $90 \%$ alcohol. The water suspension of these dried alcohol extracts were extracted using EtOAc and $n-\mathrm{BuOH}$ successively. The $n-\mathrm{BuOH}$ fraction of $\mathrm{Cl}(\mathrm{Cl}-\mathrm{B})$ was isolated by silica gel column, Sephadex LH 20 column and preparative HPLC. The isolated compounds were identified by ${ }^{1} \mathrm{H}-\mathrm{NMR},{ }^{13} \mathrm{C}-\mathrm{NMR}, \mathrm{HMBC}, \mathrm{HMQC}$ and ESI-Q-TOF MS, All the fractions and compounds isolated were evaluated on human recombinant aldose reductase (HR-AR) assay, advanced glycation end products (AGEs) formation assay and human COLO 320 DM colon cancer cells inhibitory assay.

Results: $\mathrm{Cl}-\mathrm{B}$ was found to show a significant inhibitory effect in above biological screenings. Six flavonoids and three polyphenolic acids were obtained from Cl-B. They were identified as kaempferol (1), kaempferol-3-O-[6"-O-(E)-pcoumaroyl]- $\beta$-D-glucopyranoside (2), kaempferol-3-O-[6"-O-(E)-p-coumaroyl]- $\beta$-D-galactopyranoside (3), kaempferol3-O-[2"-O-(E)-p-coumaroyl]- $\beta$-D-glucopyranoside (4), kaempferol-3-O-[2", 6"-di-O-(E)-p-coumaroyl]- $\beta$-D-glucopyranoside (5) and kaempferol-3-O-[2", 6"-di-O-(E)-p-coumaroyl]- $\beta$-D-galactopyranoside (6), casuariin (7), casuarinin (8) and castalagin (9). Compounds $2-9$ were found to show higher activity than quercetin (positive control) in the AR assay. Compounds 3-6, 8, and 9 showed stronger inhibitory effects than amino guanidine (positive control) on AGEs production. Compounds 4-6, 7, and 8 showed much higher cytotoxic activity than 5-fluorouracil (positive control) against the human COLO 320 DM colon cancer cells.
\end{abstract}

Conclusions: Our results suggest that flavonoids and polyphenolic acids possesses anti-diabetes complications and anti-cancer properties, and they were presumed to be the bioactive components of Castanea mollissima Blume.

Keywords: Anti-diabetic complications, Anti-cancer, Castanea mollissina Blume, Phenolic acids, Flavonoids

\section{Background}

Castanea mollissima Blume (family Fagaceae), with the Chinese name "Ban li" (chestnut) is widely distributed in Asian areas, such as China, Korea and Viet Nam. Chestnut fruits are highly regarded and widely consumed throughout Asia, Europe and America. Various commercial forms

\footnotetext{
*Correspondence: sypugaohy@163.com; zhanlb@dlmedu.edu.cn ${ }^{2}$ Key Laboratory of Structure-Based Drug Design \& Discovery of Ministry of Education, Shenyang Pharmaceutical University, No.103, Wenhua Road, Shenhe District, Shenyang 110016, China

${ }^{4}$ Department of Traditional Chinese Medicine, The Second Affiliated Hospital, Dalian Medical University, Dalian 116023, China

Full list of author information is available at the end of the article
}

are available. Ban li is not only used as a food product for its various nutrients, but also used as a traditional Chinese medicine, including the flowers, leaves, and twigs to have been used to treat gastroenteritis, bronchitis and regurgitation for hundreds years [1]. It is no doubt that chestnuts have considerable potential value as functional foods [2].

To increase and find much more functional values for the Chinese chestnut, our previous work on the chemical constituents and bioactivity of it resulted in the isolation of many flavonoids and phenolic acids [3-6]. In the continuous assessing its bioactivity and finding more

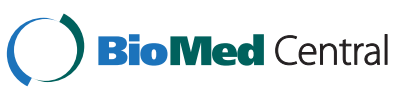

(c) 2014 Zhang et al.; licensee BioMed Central Ltd. This is an Open Access article distributed under the terms of the Creative Commons Attribution License (http://creativecommons.org/licenses/by/4.0), which permits unrestricted use, distribution, and reproduction in any medium, provided the original work is properly credited. The Creative Commons Public Domain Dedication waiver (http://creativecommons.org/publicdomain/zero/1.0/) applies to the data made available in this article unless otherwise stated. 
active agents, the anti-diabetic complications and anticancer activity of all fractions of alcohol extracts of kernels (CK), shells (CS) and involucres (CI) were evaluated on human recombinant aldose reductase (HR-AR) assay, advanced glycation end products (AGEs) formation assay and human COLO 320 DM colon cancer cells inhibitory assay in the present work, respectively.

Diabetes, as a complex metabolic disorder caused by insulin insufficiency and/or insulin dysfunction, is characterized by aberrant blood glucose and insulin levels [7]. Diabetic complications, including retinopathy, neuropathy, nephropathy, and arteriosclerosis are considered as risk factors for morbidity and death. Moreover, the diabetic patients are also susceptible to many diseases, including the cancer. For example, colorectal cancer which is the third leading cause of cancer-related death [8], is much easier happened in diabetic patient [9]. Many studies on chestnut seeds and other parts of this plant emphasised on the anti-oxidant property $[2,10]$, while, the present work were to value their anti-diabetes complications and against the connecting cancer activity, also to find the part with much functional values.

\section{Methods}

\section{Plant material}

The kernel, shells and involucres parts of C. mollissima were collected respectively in September, 2005 in Qianxi County of Hebei province, and identified by Professor Sun Qishi (College of Traditional Chinese Medicine, Shenyang Pharmaceutical University). The voucher specimens were deposited at the Key Laboratory of StructureBased Drug Design \& Discovery of Ministry of Education (No.ZB2005-026-028).

\section{Chemical and reagents}

Dibasic sodium phosphate, sodium dihydrogen phosphate, D,L-glyceraldehyde, human recombinant aldose reductase (HR-AR), AG, quercetin, critric acid monohydrate, natrium carbonicum, sodium azide, gelatin and sulphuric acid were purchased from Wako Pure Chemical Industries, Ltd. (Osaka, Japan). Sodium bicarbonate, sodium chloride, potassium dihydrogen phosphate and ethanol were supplied by Nacalai Inc. (Kyoto, Japan). Tween 20, bovine serum albumin, glucose, $O$-phenylenediamine dihydrochloride, phosphate buffered saline, fetal bovine serum (FBS), steroyl myristoyl phosphatidylcholine glycine (SMPC Gly), 3-(4, 5-dimethylthazol-2-yl)-2, 5-diphenyl tetrazolium bromidetetrazolium salt (MTT) were purchased from Sigma-Aldrich company, Ltd. (St. Louis, MO, U.S.A.). NADPH was provided by Oriental Yeast Co., Ltd. (Tokyo, Japan). Anti-AGE antibody and goat anti-mouse IgG, HRP conjugate-secondary antibody Millipore (Merck U.S.A) were purchased from Transgenic Inc. (Hyogo, Japan). Silica gel $60 \mathrm{~F}_{254}$ TLC plates
(Merck, U.S.A), silica gel $60 \mathrm{~N}(100-200 \mu \mathrm{m})$ and ODS were purchased from Kanto Chemical (Tokyo, Japan). DMSO was purchased from Wako Pure Chemical Industries, Ltd (Japan). Kaempferol-3-O- $\beta$-D-glucopyranoside was isolated from liquorice, and was identified by NMR and MS data [11].

\section{Instrument}

Analysis HPLC was carried out on Waters 510 with Waters 484 detector (waters, USA) using a kromasil C18 column (4.6 $200 \mathrm{~mm}$, Rainbow). Preparative HPLC was carried out on JASCO PU-2087 with JASCO PU-2075 detector (JASCO, Japan) using a HiQ sil C18 column $(10 \times 200 \mathrm{~mm}$, YATECH). ESI-MS spectra were recorded on a Bruker esquire 2000 mass spectrometer. NMR spectra $\left({ }^{1} \mathrm{H}-,{ }^{13} \mathrm{C}-\mathrm{NMR}, \mathrm{HMBC}\right.$ and NOESY $)$ were measured and recorded on Bruker AVANCE 600 (Bruker, Newark, DE) in DMSO using TMS as internal standard. Infrared (IR) spectra were measured on a Fourier transform infrared spectrometer (IFS-55; Bruker).

\section{HR-AR assay}

Aldose reductase activities of samples were assayed spectrophotometrically by determining the decrease in NADPH concentration at $340 \mathrm{~nm}$ in a UV-2201 Pharma Spec UV-vis spectrophotometer (Shimadzu, Japan). The reaction mixture contained $0.14 \mathrm{M}$ phosphate buffer $\mathrm{pH}$ 6.2, $700 \mu \mathrm{L} ; 0.15 \mathrm{mM}$ NADPH, $100 \mu \mathrm{L} ; 3 \times 10^{-2}$ units $/ \mathrm{mL}$ AR, $100 \mu \mathrm{L} ; 10 \mathrm{mM}$ D,L-glyceraldehyde, $3.3 \mathrm{mg} / \mathrm{mL}$ extracts or $1 \mathrm{mg} / \mathrm{mL}$ compounds in DMSO, $3 \mu \mathrm{L}$, in a total volume of $1 \mathrm{~mL}$ with a final concentration of $10 \mu \mathrm{g} / \mathrm{mL}$ for extracts and $3.3 \mu \mathrm{g} / \mathrm{mL}$ for compounds. The reference blank contained all of the above reagents, and buffer instead of AR, to correct for nonspecific reduction of NADPH. The control had only the sample solvent instead of the sample to correct for reduction of NADPH without inhibitor of AR (ARI). The reaction was initiated by the addition of substrate, and it was monitored spectrophotometrically for $3 \mathrm{~min}$. All values were averages of three independent experiments [12]. The inhibition ratio of AR was calculated by following equation:

$$
\text { Inhibition } \begin{aligned}
(\%)= & {\left[1-\left(\Delta \mathrm{ABS}_{\text {sample }}-\Delta \mathrm{ABS}_{\text {blank }}\right)\right.} \\
& \left.\div\left(\Delta \mathrm{ABS}_{\text {control }}-\Delta \mathrm{ABS}_{\text {blank }}\right)\right] \times 100
\end{aligned}
$$

\section{Inhibit formation of AGEs assay}

The AGEs reaction solution $(200 \mu \mathrm{L})$ was composed of $16 \mathrm{mg} / \mathrm{mL}$ bovine serum albumin, $50 \mu \mathrm{L} ; 0.1 \mathrm{M}$ sodium phosphate buffer ( $\mathrm{pH} 7.4$ ), $96 \mu \mathrm{L} ; 144 \mathrm{mg} / \mathrm{mL}$ glucose, $50 \mu \mathrm{L} ; 0.5 \mathrm{mg} / \mathrm{mL}$ sample in DMSO, $4 \mu \mathrm{L}$ with a final concentration of $10 \mu \mathrm{g} / \mathrm{mL}$. The sample blank, control solvent and blank solvent contained all of the above 
reagents, except sample solvent with buffer instead of glucose in the sample blank, sample solvent instead of sample in the control solvent, and sample solvent instead of sample, buffer instead of glucose in the blank solvent. After incubating at $37^{\circ} \mathrm{C}$ for 7 days, the amounts of AGE products were determined by enzyme-linked immunosorbent assay (ELISA) [13]. Noncompetitive ELISA assays were performed at room temperature. Each well was incubated for $1 \mathrm{~h}$ with $0.1 \mathrm{~mL}$ of an AGE sample to be tested or its corresponding control sample in $50 \mathrm{mM}$ carbonate buffer ( $\mathrm{pH}$ 9.7) and washed three times with washing buffer (phosphate-buffered saline containing $0.05 \%$ Tween 20). Each well was then blocked for $1 \mathrm{~h}$ with $0.2 \mathrm{~mL}$ of $2.5 \%$ gelatin in $5 \mathrm{mM}$ carbonate buffer ( $\mathrm{pH}$ 9.7). Each well was washed three times with washing buffer and incubated for $1 \mathrm{~h}$ with $0.1 \mathrm{~mL}$ of antiAGE antibody $(50 \mathrm{ng} / \mathrm{mL})$. Wells were then washed three times with washing buffer and incubated for $1 \mathrm{~h}$ with $0.1 \mathrm{~mL}$ of goat anti-mouse IgG, HRP conjugatesecondary antibody (Millipore, Merck) and then washed three times, followed by reaction with $o$-phenylenediamine dihydrochloride and hydrogen peroxide mixture. The reaction was terminated by adding $1 \mathrm{M}$ sulfuric acid, and the absorbance at $492 \mathrm{~nm}$ was read on a micro-plate reader (MPR A4i II TOSOH). All values are averages of three independent experiments, each done in triplicate [14]. The percentage inhibition of AGE production was calculated by the following equation:

$$
\begin{aligned}
\text { Inhibition }(\%)= & {\left[1-\left(\mathrm{A}_{\text {sample }}-\mathrm{A}_{\text {sample blank }}\right)\right.} \\
& \left.\div\left(\mathrm{A}_{\text {control solvent }}-\mathrm{A}_{\text {blank solvent }}\right)\right] \times 100
\end{aligned}
$$

\section{Cytotoxic assay}

Human COLO 320 DM colon cancer cells (passage-10) were maintained in RPMI-1640 medium supplemented with $10 \% \mathrm{FBS}$ and SMPC Gly at $37^{\circ} \mathrm{C}$ in an incubator. The cytotoxicity was determined by the MTT method. Briefly, exponentially growing cells, $100 \mu \mathrm{l}$, were attached at $5 \times 10^{4}$ cells/well, in 96-well plates, and the cellular viability was determined after $24 \mathrm{~h}, 48 \mathrm{~h}$, and $72 \mathrm{~h}$ administration of the extractions $(100 \mu \mathrm{g} / \mathrm{mL})$ $100 \mu \mathrm{L}$. Cells were incubated with MTT tetrazolium salt for $1 \mathrm{~h}$ at $37^{\circ} \mathrm{C}$, and the formation of formazan was measured by a microplate reader (MPR A4iIITOSOH). All values are averages of two independent experiments, each done in triplicate. The percentage inhibition of cell growth was calculated by the following equation:

$$
\text { Inhibition }(\%)=100-[(A 1 / A 0) \times 100]
$$

Where A0 is the absorbance of the control at $500 \mathrm{~nm}$ after incubations, and $\mathrm{A} 1$ is the absorbance in the presence of the samples. The study was performed in accordance with the Declaration of Helsinki.

\section{Extraction and isolation}

Air-dried three parts of kernels $6 \mathrm{~kg}$ (CK), shells $5 \mathrm{~kg}$ (CS) and involucres $10 \mathrm{~kg}(\mathrm{CI})$ of were C. mollissima crushed and extracted with ethanol-water (90:10, v/v) (CK: $36 \mathrm{~L}$; CS: $30 \mathrm{~L}$ and CI: $60 \mathrm{~L}$ ) for three times (for $2 \mathrm{~h} \times 3$ ) respectively, then, the solutions were concentrated under reduced pressure using a rotary evaporator less than $40^{\circ} \mathrm{C}$, to give extracts (CK 953.2 g, 15.89\%; CS 257.4 g, 5.15\%; CI $305.6 \mathrm{~g}, 3.06 \%$ ), respectively. The extracts were suspended in distilled water (CK: $6 \mathrm{~L}, \mathrm{CS}: 5 \mathrm{~L}$, and CI: $10 \mathrm{~L}$ ) and partitioned successively with the same volume EtOAc and $n$ $\mathrm{BuOH}$ successively. After removing organic solvents, three EtOAC soluble fractions CK-A (30.2 g, 0.50\%), CS-A (35.9 g, 0.72\%), CI-A (95.7 g, 0.96\%) and three $n-\mathrm{BuOH}$ soluble fractions CK-B (61.5 g, 1.03\%), CS-B (21.8 g, $0.44 \%)$, CI-B (62.1 g, 0.62\%), along with three aqueous parts CK-W (811.9 g, 13.53\%), CS-W (140.6 g, 2.81\%), CIW $(125.9 \mathrm{~g}, 1.26 \%)$ were obtained. Each part was vacuumpacked and stored at $-20^{\circ} \mathrm{C}$ until use.

Part of CI-B (60 g) was taken and mixed with $180 \mathrm{~g}$ silica then applied to a $85 \mathrm{~cm} \times 10 \mathrm{~cm}$ (inside diameter) silica gel column and eluted with different ratios of EtOAc and $\mathrm{MeOH}(10: 1,10: 2,10: 4,10: 6,10: 8,1: 1,1: 5,0: 1)$ to give 10 fractions based on the results of TLC experiment. Fraction 2 (9.75 g) eluted with EtOAc-MeOH (10:1) was further separated into 12 sub-fractions by a $60 \mathrm{~cm} \times 7 \mathrm{~cm}$ (inside diameter) silica gel column based on the results of TLC experiment. Sub-fraction 7 (1.25 g) eluted with HexaneEtOAc (1:3) was purified by preparative HPLC with $\mathrm{MeOH}-\mathrm{H}_{2} \mathrm{O}(60: 40 \mathrm{v} / \mathrm{v}, 1.5 \mathrm{~mL} / \mathrm{min})$ on a $\mathrm{C}_{18}$-MS-II-waters column $(10 \times 250 \mathrm{~mm})$ to yield compound $\mathbf{1}(30 \mathrm{mg})$. Subfraction 9 (0.87 g) eluted with Hexane-EtOAc (3:1) was purified by a $40 \mathrm{~cm} \times 6 \mathrm{~cm}$ (inside diameter) ODS gel column with $\mathrm{MeOH}-\mathrm{H}_{2} \mathrm{O}$ (30:70, 40:60, 50:50, 60:40, 100:0 v/v) and preparative HPLC with $\mathrm{MeOH}-\mathrm{H}_{2} \mathrm{O}$ $(55: 45 \mathrm{v} / \mathrm{v}, 1.5 \mathrm{~mL} / \mathrm{min})$ on a $\mathrm{C}_{18}$-MS-waters column $(10 \times 250 \mathrm{~mm})$ to yield compound $5(30 \mathrm{mg})$ and compound $6(15 \mathrm{mg})$. Sub-fraction $10(1.50 \mathrm{~g})$ eluted with Hexane-EtOAc (6:1) was purified by preparative HPLC with $\mathrm{MeOH}-\mathrm{H}_{2} \mathrm{O}(55: 45 \mathrm{v} / \mathrm{v})$ on a $\mathrm{C}_{18}$-MS-waters column $(10 \times 250 \mathrm{~mm})$ to yield compounds $2(7 \mathrm{mg}), 3(30 \mathrm{mg})$ and $4(25 \mathrm{mg})$. Fraction $3(26.0 \mathrm{~g})$ eluted with EtOAc$\mathrm{MeOH}(10: 1)$ was further separated into 8 sub-fractions by a $80 \mathrm{~cm} \times 9 \mathrm{~cm}$ (inside diameter) silica gel column. Compounds 7-9 was obtained from sub-fraction 5 (5.36 g) which was eluted with Hexane-EtOAc (6:1) by a $50 \mathrm{~cm} \times 7 \mathrm{~cm}$ (inside diameter) ODS gel column with $\mathrm{MeOH}-\mathrm{H}_{2} \mathrm{O}(30: 70,40: 60,50: 50,60: 40,100: 0 \mathrm{v} / \mathrm{v})$ and an $80 \mathrm{~cm} \times 4 \mathrm{~cm}$ (inside diameter) Sephadex LH-20 column with $\mathrm{MeOH}-\mathrm{H}_{2} \mathrm{O}(50: 50 \mathrm{v} / \mathrm{v})$. Structures of compounds 1-9 were shown in Figure 1. 

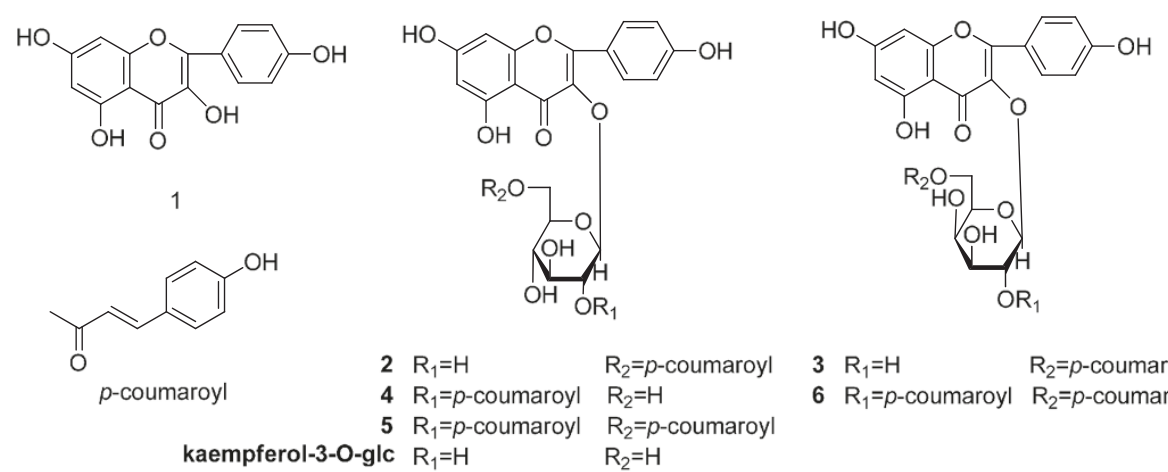

$3 \mathrm{R}_{1}=\mathrm{H} \quad \mathrm{R}_{2}=p$-coumaroyl

$6 \mathrm{R}_{1}=p$-coumaroyl $\mathrm{R}_{2}=p$-coumaroyl

kaempferol-3-O-glc $\mathrm{R}_{1}=\mathrm{H}$
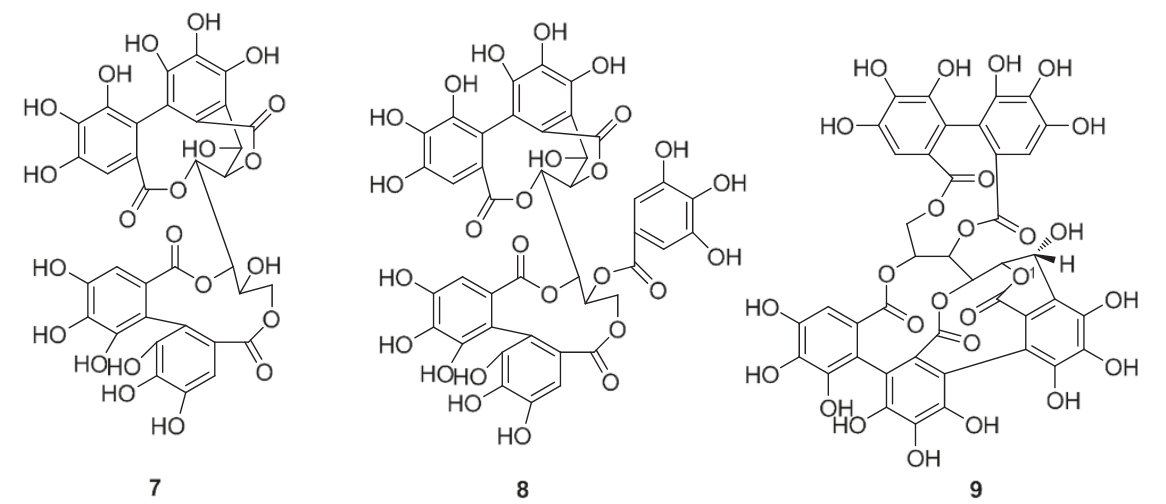

Figure 1 The structures of compounds 1-9.

\section{Hydrolysis of compound 6}

Compound $6(1.0 \mathrm{mg})$ was dissolved in $\mathrm{MeOH}(9 \mathrm{~mL})$ containing $\mathrm{HCl}(1 \mathrm{~mL})$ and refluxed on a heated $\left(80^{\circ} \mathrm{C}\right)$ water bath for $3 \mathrm{~h}$. After cooling, the reaction mixture was concentrated and dried under reduced pressure, then analyzed by TLC using the solvent system $\mathrm{CHCl}_{3}-$ $\mathrm{MeOH}-\mathrm{H}_{2} \mathrm{O}$ (20:10:1) for identification of the complete reaction. The dried residues dissolved in EtOAc, and partitioned with water. The water solution was analyzed and subjected to HPLC with optical detector using a Kaseisorb LC NH $\mathrm{H}_{2}-60-5$ column $(4.6 \mathrm{~mm}$ i.d. $\times 250 \mathrm{~nm}$ ) with $\mathrm{CH}_{3} \mathrm{CN}-\mathrm{H}_{2} \mathrm{O}$ (v:v $=85: 15$, Flow: $0.8 \mathrm{~mL} / \mathrm{min}$ ) as the mobile phase. Identification of D-galactose was carried out by comparison of the retention time $(13.2 \mathrm{~min})$ and OD $(+)$ with authentic samples.

\section{Results}

\section{Structural determination of compound 6}

Kaempferol-3-O-[2", 6 "-di- $O-(E)$ - $p$-coumaroyl]- $\beta$-D-galactopyranoside (6) was obtained as a yellow powder with a negative optical rotation. Its structure identification was achieved by mass and NMR spectroscopy (Additional files 1, 2, 3, 4, 5, 6 and 7), including two dimensional correlated NMR (NOESY, HMQC, HMBC). Its molecular formula was established as $\mathrm{C}_{39} \mathrm{H}_{32} \mathrm{O}_{15}$ by means of ESIQ-TOF MS which showed an $[\mathrm{M}+\mathrm{H}]^{+}$ion peak at $\mathrm{m} / z$
741.1824 (calcd 741.1819 for $\mathrm{C}_{39} \mathrm{H}_{33} \mathrm{O}_{15}$ ). ${ }^{1} \mathrm{H}$ - and ${ }^{13} \mathrm{C}$ NMR spectral data of 6 (Table 1) showed the presence of a kaempferol residue, two $p$-coumaroyl groups and one sugar unit. The HMBC spectrum (Figure 2) clarified the attachment of the sugar group at $\mathrm{C}-3$ position based on a correlation between the anomeric proton signal at $\delta_{\mathrm{H}}$ 5.54 (d, $J=7.6)$ with $\mathrm{C}-3$ (104.65). The attachments for two $p$-coumaroyl groups at $\mathrm{C}-2$ " and $\mathrm{C}-6$ " were based on correlations between $\delta_{\mathrm{H}} 5.38(\mathrm{dd}, J=9.6,8.0, \mathrm{H}-2$ ") with $\delta_{\mathrm{C}} 168.23\left(\mathrm{C}-9{ }^{\prime \prime}\right)$, and, $\delta_{\mathrm{H}} 4.19,4.36$ (dd, 11.6, H6") with $\delta_{\mathrm{C}} 168.21\left(\mathrm{C}-9^{\prime \prime \prime}\right)$, respectively. The trans form configurations for their double bonds at C-7"', 8 "' and C$7^{\prime \prime \prime}, 8^{\prime \prime \prime}$ positions were confirmed by the coupling constant values $15.6 \mathrm{~Hz}$ for H-7"' and H-7"'" [15]. The sugar unit was identified as galactose after the sample was dealt with the acid hydrolysis and compared the $t_{R}$ with the authentic sample by HPLC analysis using a Kaseisorb LC $\mathrm{NH}_{2}-60-5$ column $\left(4.6 \mathrm{~mm}\right.$ i.d. $\times 250 \mathrm{~nm}$ ), $\mathrm{CH}_{3} \mathrm{CN}-\mathrm{H}_{2} \mathrm{O}$ as the mobile phase. Compound $\mathbf{6}$ was identified as kaempferol-3-O-[2", 6"-di- $O-(E)$ - $p$-coumaroyl]- $\beta$-D-galactopyranoside at finally.

\section{Effects of fractions and compounds in HR-AR, AGEs and Cytotoxic assay}

All crude materials and soluble fractions (EtOAc, $n$ $\mathrm{BuOH}$ and water part) of kernels (CK, CK-A, CK-B, CK- 
Table 1 NMR spectra data of compound 6 in $\mathrm{CD}_{3} \mathrm{OD}$

\begin{tabular}{|c|c|c|c|}
\hline No. & ${ }^{1} \mathrm{H}-\mathrm{NMR}$ & ${ }^{13}$ C-NMR & HMBC \\
\hline 2 & & $158.17 \mathrm{~s}$ & \\
\hline 3 & & $134.58 \mathrm{~s}$ & \\
\hline 4 & & $179.00 \mathrm{~s}$ & \\
\hline 5 & & $162.69 \mathrm{~s}$ & \\
\hline 6 & $6.06(1 \mathrm{H}, \mathrm{br} . \mathrm{s})$ & $99.79 \mathrm{~d}$ & $5,7,8,10$ \\
\hline 7 & & $165.32 \mathrm{~s}$ & \\
\hline 8 & $6.25(1 \mathrm{H}, \mathrm{d}, 1.6)$ & $94.54 \mathrm{~d}$ & $6,7,9,10$ \\
\hline 9 & & $157.96 \mathrm{~s}$ & \\
\hline 10 & & $105.55 \mathrm{~s}$ & \\
\hline $1^{\prime}$ & & $122.52 \mathrm{~s}$ & \\
\hline $2^{\prime}, 6^{\prime}$ & $7.96(2 \mathrm{H}, \mathrm{d}, 8.8)$ & $131.95 \mathrm{~d}$ & $2,4^{\prime}$ \\
\hline $3^{\prime}, 5^{\prime}$ & $6.87(2 \mathrm{H}, \mathrm{d}, 8.8)$ & $116.10 \mathrm{~d}$ & $4^{\prime}, 1^{\prime}$ \\
\hline $4^{\prime}$ & & $161.13 \mathrm{~s}$ & \\
\hline $1^{\prime \prime}$ & $5.54(1 \mathrm{H}, \mathrm{d}, 8.0)$ & $100.85 d$ & $3,2^{\prime \prime}$ \\
\hline $2^{\prime \prime}$ & $5.38(1 \mathrm{H}, \mathrm{dd}, 9.6,8.0)$ & $73.98 \mathrm{~d}$ & $1^{\prime \prime}, 3^{\prime \prime}, 9^{\prime \prime \prime}$ \\
\hline $3^{\prime \prime}$ & $3.82(1 \mathrm{H}, \mathrm{m})$ & $73.13 d$ & $2^{\prime \prime}$ \\
\hline $4^{\prime \prime}$ & $3.88(1 \mathrm{H}, \mathrm{m})$ & $70.53 d$ & $2^{\prime \prime}$ \\
\hline $5^{\prime \prime}$ & $3.85(1 \mathrm{H}, \mathrm{m})$ & $74.90 \mathrm{~d}$ & \\
\hline \multirow[t]{2}{*}{$6^{\prime \prime}$} & $4.19(1 \mathrm{H}, \mathrm{dd}, 11.6)$ & $64.15 \mathrm{t}$ & $9^{\prime \prime \prime \prime}$ \\
\hline & $4.36(1 \mathrm{H}, \mathrm{dd}, 11.6)$ & & \\
\hline $1^{\prime \prime \prime}$ & & $127.09 \mathrm{~s}$ & \\
\hline $2^{\prime \prime \prime}, 6^{\prime \prime \prime}$ & $7.46(2 \mathrm{H}, \mathrm{d}, 8.4)$ & $131.00 \mathrm{~d}$ & $4^{\prime \prime \prime}, 7^{\prime \prime \prime}$ \\
\hline $3^{\prime \prime \prime}, 5^{\prime \prime \prime}$ & $6.79(2 \mathrm{H}, \mathrm{d}, 8.4)$ & $116.59 \mathrm{~d}$ & $1^{\prime \prime \prime}, 4^{\prime \prime \prime}$ \\
\hline $4^{\prime \prime \prime}$ & & $160.94 \mathrm{~s}$ & \\
\hline $7^{\prime \prime \prime}$ & $7.69(1 \mathrm{H}, \mathrm{d}, 15.6)$ & $146.68 d$ & $6^{\prime \prime \prime}, 8^{\prime \prime \prime}, 9^{\prime \prime \prime}$ \\
\hline $8^{\prime \prime \prime}$ & $6.40(1 \mathrm{H}, \mathrm{d}, 15.6)$ & $115.12 \mathrm{~d}$ & $1^{\prime \prime \prime}, 9^{\prime \prime \prime}$ \\
\hline $9^{\prime \prime \prime}$ & & $168.23 \mathrm{~s}$ & \\
\hline $1^{\prime \prime \prime \prime}$ & & $126.87 \mathrm{~s}$ & \\
\hline $2^{\prime \prime \prime \prime}, 6^{\prime \prime \prime \prime}$ & $7.27(2 \mathrm{H}, \mathrm{d}, 8.4)$ & $130.92 \mathrm{~d}$ & $4^{\prime \prime \prime \prime}, 7^{\prime \prime \prime \prime}$ \\
\hline $3^{\prime \prime \prime \prime}, 5^{\prime \prime \prime \prime}$ & $6.79(2 \mathrm{H}, \mathrm{d}, 8.4)$ & $116.59 \mathrm{~d}$ & $1^{\prime \prime \prime \prime}, 4^{\prime \prime \prime \prime}$ \\
\hline $4^{\prime \prime \prime \prime}$ & & $160.87 \mathrm{~s}$ & \\
\hline $7^{\prime \prime \prime \prime}$ & $7.40(1 \mathrm{H}, \mathrm{d}, 15.6)$ & $146.35 d$ & $6^{\prime \prime \prime \prime}, 8^{\prime \prime \prime \prime}, 9^{\prime \prime \prime \prime}$ \\
\hline $8^{\prime \prime \prime \prime}$ & $6.05(1 \mathrm{H}, \mathrm{d}, 15.6)$ & $114.43 d$ & $9^{\prime \prime \prime \prime}$ \\
\hline $9^{\prime \prime \prime \prime}$ & & $168.21 \mathrm{~s}$ & \\
\hline
\end{tabular}

W), shells (CS, CS-A, CS-B, CS-W) and involucres (CI, CI-A, CI-B, CI-W) were evaluated on AR, AGEs and the growth inhibitory activity aginst human COLO $320 \mathrm{DM}$ colon cancer cells. Among these fractions, the $n-\mathrm{BuOH}$ soluble fraction of involucres (CI-B) was found to be significantly more active than others (Figure 3 ), and its inhibitory rates were $88.6 \pm 2.3 \%$ in $\mathrm{AR}, 77.0 \pm 5.8 \%$ in AGEs, and $81.3 \pm 3.7 \%$ in cytotoxic assay respectively. Effective compounds from CI-B were further studied. Six flavonoids, kaempferol (1), kaempferol-3-O-[6"-O-(E)-pcoumaroyl]- $\beta$-D-glucopyranoside (2), kaempferol-3-O-

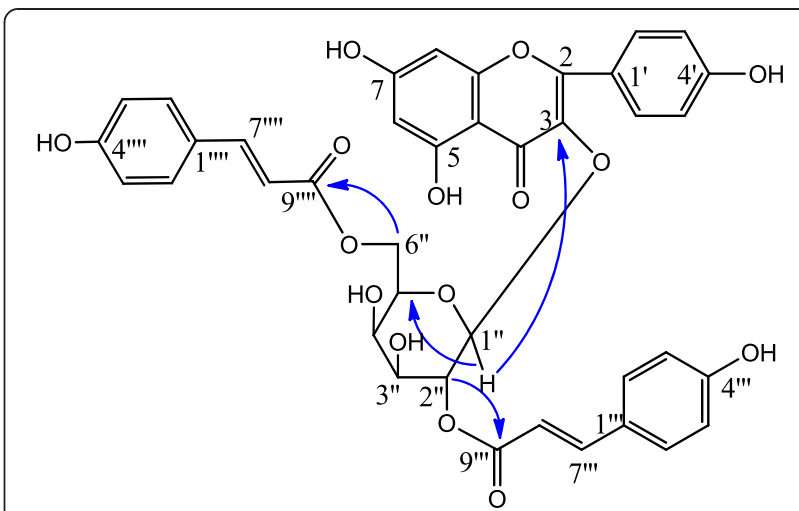

Figure 2 Key HMBC correlations of compound 6.

[6"-O-(E)-p-coumaroyl]- $\beta$-D-galatopyranoside (3), kaempferol-3-O-[2"-O- $(E)$ - $p$-coumaroyl] $-\beta$-D-glucopyranoside (4), kaempferol-3-O-[2", 6 "-di-O-(E)-p-coumaroyl]- $\beta$-Dglucopyranoside (5), kaempferol-3-O-[2", 6"-di-O- $(E)-p$ coumaroyl]- $\beta$-D-galactopyranoside (6), and three polyphenolic acids, casuariin (7), casuarinin (8), and castalagin (9) were obtained. Structures of compounds (see Figure 1) were elucidated by the spectroscopic methods, including UV, ESI-MS, NMR experiments, and comparing their spectral data with reported in the references $[3,16,17]$. The inhibitory activities of compounds 1-9 were shown in Table 2.

Physicochemical data of compounds 2-9

kaempferol-3-O-[6"-O-(E)-p-coumaroyl]- $\beta$-D-glucopyranoside

(2). Yellow powder; m.p.223-225 $\mathrm{C} ;[\alpha]_{\mathrm{D}}^{23}-39.0^{\circ}$ (c 0.05, MeOH). UV $\lambda_{\max }(\mathrm{MeOH}) \mathrm{nm}(\log \varepsilon): 316(2.25)$. FAB-MS m/z: $595[\mathrm{M}+\mathrm{H}]^{+}$and $593[\mathrm{M}-\mathrm{H}]^{-}$. ${ }^{1} \mathrm{H}-\mathrm{NMR}$ and ${ }^{13} \mathrm{C}$-NMR spectra data see Table 3 .

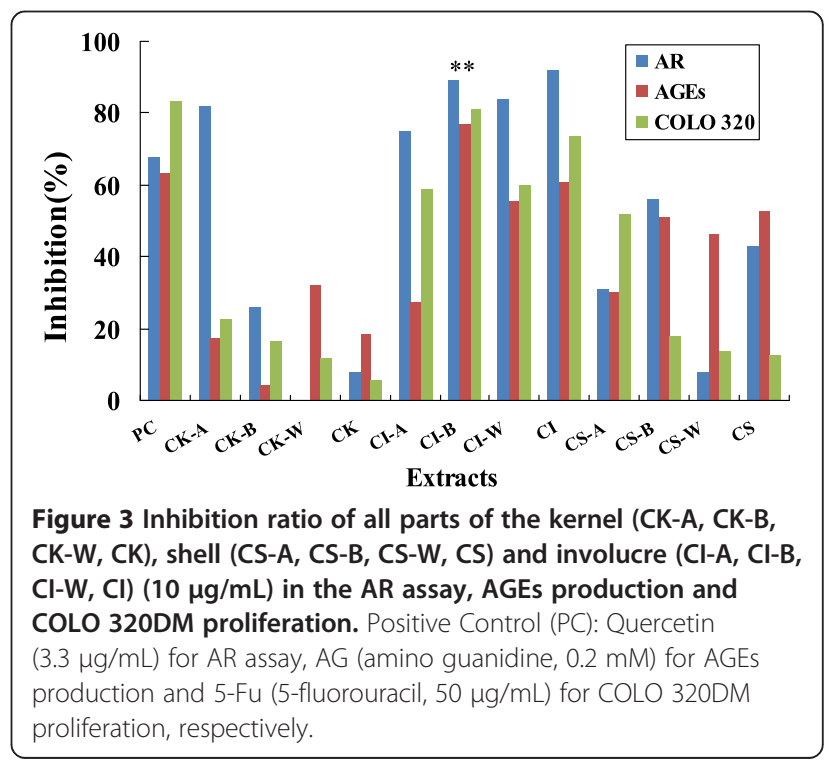




\begin{tabular}{|c|c|c|c|}
\hline \multirow[t]{2}{*}{ Compounds } & \multicolumn{3}{|c|}{$\mathrm{IC}_{50}(\mu \mathrm{M})$} \\
\hline & AR & AGEs & COLO 320 \\
\hline 1 & $14.83 \pm 0.28^{\triangle}$ & $57.06 \pm 7.54$ & $>100$ \\
\hline 2 & $2.39 \pm 0.20^{* * *}$ & $28.73 \pm 2.96$ & $24.10 \pm 3.26$ \\
\hline 3 & $1.83 \pm 0.02^{* * *}$ & $8.48 \pm 1.43^{*}$ & $22.56 \pm 4.51^{\triangle}$ \\
\hline 4 & $1.22 \pm 0.06^{* * *}$ & $5.58 \pm 0.48^{* * *}$ & $3.53 \pm 0.42^{* *}$ \\
\hline 5 & $0.43 \pm 0.06^{* * *}$ & $3.27 \pm 0.09^{* * *}$ & $2.86 \pm 0.39^{* *}$ \\
\hline 6 & $0.63 \pm 0.12^{* * *}$ & $0.75 \pm 0.21^{* * *}$ & $2.43 \pm 1.01^{* *}$ \\
\hline kaempferol-3-O-glc & $10.40 \pm 0.18^{* *}$ & $42.64 \pm 3.73$ & $58.84 \pm 3.26$ \\
\hline 7 & $6.33 \pm 1.10^{* *}$ & $12.69 \pm 5.17^{\Delta \Delta}$ & $10.07 \pm 1.84^{*}$ \\
\hline 8 & $0.46 \pm 0.01^{* * *}$ & $4.43 \pm 0.36^{* * *}$ & $5.49 \pm 0.23^{*}$ \\
\hline 9 & $1.73 \pm 0.14^{* * *}$ & $5.21 \pm 0.75^{* * *}$ & $3.67 \pm 0.09^{* *}$ \\
\hline Qu & $12.35 \pm 1.06$ & & \\
\hline$A G$ & & $10.68 \pm 1.06$ & \\
\hline $5-\mathrm{Fu}$ & & & $8.79 \pm 2.57$ \\
\hline
\end{tabular}

Data represent mean \pm S.E.M. $(n=3)$. IC $C_{50}$ of Compounds $<I C_{50}$ of Positive Control (Qu, AG, 5-Fu): ${ }^{* * *} p<0.01$ vs Positive Control, ${ }^{* *} p<0.05$ vs Positive Control, ${ }^{*} p>0.05$ vs Positive Control; IC $C_{50}$ of Compounds $>I C_{50}$ of Positive Control (Qu, AG, 5-Fu): ${ }^{\Delta} p>0.05$ vs Positive Control. ${ }^{\Delta} p>0.01$ vs Positive Control.

kaempferol-3-O-[6"-O- $(E)-p$-coumaroyl]- $\beta$-D-galatopyranoside

(3). Yellow powder (40.0 mg). m.p.245-247 ${ }^{\circ} \mathrm{C} \cdot[\alpha]_{\mathrm{D}}^{22}-43.0^{\circ}$ (c $0.05, \mathrm{MeOH})$. UV $\lambda_{\max }(\mathrm{MeOH}) \mathrm{nm}(\log \varepsilon): 318$ (2.09). FAB-MS m/z: $595[\mathrm{M}+\mathrm{H}]^{+}$and $593[\mathrm{M}-\mathrm{H}]^{-} .{ }^{1} \mathrm{H}-\mathrm{NMR}$ and ${ }^{13} \mathrm{C}$-NMR spectra data see Table 3 .

kaempferol-3-O-[2"-O-(E)-p-coumaroyl]- $\beta$-D-glucopyranoside

(4). Yellow powder. m.p. $245-247^{\circ} \mathrm{C} .[\alpha]_{\mathrm{D}}^{20}-74.1^{\circ}$ (c 0.13, $\mathrm{MeOH})$. UV $\lambda_{\max }(\mathrm{MeOH}) \mathrm{nm}(\log \varepsilon): 316$ (2.74). FABMS m/z: $595[\mathrm{M}+\mathrm{H}]^{+}$and $593[\mathrm{M}-\mathrm{H}]^{-} \cdot{ }^{1} \mathrm{H}-\mathrm{NMR}$ and ${ }^{13} \mathrm{C}$-NMR spectra data see Table 3.

kaempferol-3-O-[2", 6"-di-O- $(E)$ - $p$-coumaroyl]- $\beta$-D-glucopyranoside

(5). Yellow powder. m.p. $196-199^{\circ} \mathrm{C} .[\alpha]_{\mathrm{D}}^{22}-119.6^{\circ}$ (c 0.8, $\mathrm{MeOH})$. UV $\lambda_{\max }(\mathrm{MeOH}) \mathrm{nm}(\log \varepsilon): 330$ (2.38). FABMS m/z: $741[\mathrm{M}+\mathrm{H}]]^{+} \cdot{ }^{1} \mathrm{H}-\mathrm{NMR}$ and ${ }^{13} \mathrm{C}-\mathrm{NMR}$ spectra data see Table 3 .

kaempferol-3-O-[2", 6"-di-O- $(E)$-p-coumaroyl]- $\beta$-D-galactopyranoside

(6). Yellow powder. m.p.200-203 ${ }^{\circ} \mathrm{C}$. $[\alpha]_{\mathrm{D}}^{22}-62.2^{\circ}$ (c 0.1 , $\mathrm{MeOH}) . \mathrm{UV}_{\max }(\mathrm{MeOH}) \mathrm{nm}(\log \varepsilon): 323$ (2.51); FABMS m/z: $741[\mathrm{M}+\mathrm{H}]^{+}, 739[\mathrm{M}-\mathrm{H}]^{-}$and $763[\mathrm{M}+\mathrm{Na}]^{+}$; ESI-Q-TOF MS $m / z$ 741.1824 $[\mathrm{M}+\mathrm{H}]^{+}($calcd 741.1819 for $\left.\mathrm{C}_{39} \mathrm{H}_{33} \mathrm{O}_{15}\right)$. ${ }^{1} \mathrm{H}-\mathrm{NMR},{ }^{13} \mathrm{C}-\mathrm{NMR}$ and $2 \mathrm{D}$-NMR spectra data see Table 1 .

casuariin

(7). Off-white amorphous powder. m.p.245-248 ${ }^{\circ} \mathrm{C}$. FAB-MS m/z: $783[\mathrm{M}-\mathrm{H}]^{-} .{ }^{1} \mathrm{H}-\mathrm{NMR}\left(\left(\mathrm{CD}_{3}\right)_{2} \mathrm{CO}+\mathrm{D}_{2} \mathrm{O}\right)$ $\delta: 6.81(1 \mathrm{H}, \mathrm{s}), 6.62(1 \mathrm{H}, \mathrm{s}), 6.47(1 \mathrm{H}, \mathrm{s}), 5.64(1 \mathrm{H}, \mathrm{d}, \mathrm{J}=$
$4.8 \mathrm{~Hz}), 4.72(1 \mathrm{H}, \mathrm{m}), 5.51(1 \mathrm{H}, \mathrm{m}), 5.13(1 \mathrm{H}, \mathrm{dd}, \mathrm{J}=8.4$, $3.2 \mathrm{~Hz}), 4.18(1 \mathrm{H}, \mathrm{m}), 3.93(1 \mathrm{H}, \mathrm{d}, \mathrm{J}=12.0 \mathrm{~Hz}), 4.72(1 \mathrm{H}$, d, J = 11.8 Hz). ${ }^{13} \mathrm{C}-\mathrm{NMR}\left(\left(\mathrm{CD}_{3}\right)_{2} \mathrm{CO}+\mathrm{D}_{2} \mathrm{O}\right) \delta: 115.9(\mathrm{~s})$, 115.6 (s), 115.6 (s), 114.5 (s), 126.8 (s), 126.5 (s), 124.6 (s), 119.4 (s), 116.6 (d), 108.2 (d), 106.6 (d), 104.8 (d), 145.5 (s), 145.2 (s), 144.7 (s), 144.6 (s), 143.9 (s), 143.8 (s), 143.2 (s), 143.0 (s), 138.3 (s), 136.2 (s), 135.2 (s), 134.4 (s), 169.8 (s), 169.3 (s), 168.3 (s), 165.3 (s), 66.6 (d), $76.6(d), 70.3(d), 76.5(d), 67.8(d), 67.9(q)$.

casuarinin

(8). Off-white amorphous powder. m.p.244-246 ${ }^{\circ} \mathrm{C}$. FAB-MS m/z: $935[\mathrm{M}-\mathrm{H}]^{-} .{ }^{1} \mathrm{H}-\mathrm{NMR}\left(\left(\mathrm{CD}_{3}\right)_{2} \mathrm{C} \mathrm{O}+\mathrm{D}_{2} \mathrm{O}\right)$ $\delta: 6.91(1 \mathrm{H}, \mathrm{s}), 6.64(1 \mathrm{H}, \mathrm{s}), 6.57(1 \mathrm{H}, \mathrm{s}), 7.16(2 \mathrm{H}, \mathrm{s})$, $5.70(1 \mathrm{H}, \mathrm{d}, \mathrm{J}=4.8 \mathrm{~Hz}), 4.73(1 \mathrm{H}, \mathrm{m}), 5.47(1 \mathrm{H}, \mathrm{m}), 5.52$ $(1 \mathrm{H}, \mathrm{d}, \mathrm{J}=8.8 \mathrm{~Hz}), 5.40(1 \mathrm{H}, \mathrm{m}), 4.20(1 \mathrm{H}, \mathrm{d}, \mathrm{J}=$ $13.2 \mathrm{~Hz}), 4.89(1 \mathrm{H}, \mathrm{m}) .{ }^{13} \mathrm{C}-\mathrm{NMR}\left(\left(\mathrm{CD}_{3}\right)_{2} \mathrm{CO}+\mathrm{D}_{2} \mathrm{O}\right) \delta$ : 115.6 (s), 115.4 (s), 115.3 (s), 114.3 (s), 126.3 (s), 125.8 (s), 123.8 (s), 119.1 (s), 117.0 (s), 108.2 (d), 106.7 (d), 105.0 (d), 145.2 (s), 145.1 (s), 144.6 (s), 144.6 (s), 143.9 (s), $143.2(\mathrm{~s}), 142.7(\mathrm{~s}), 138.3(\mathrm{~s}), 136.4(\mathrm{~s}), 135.3(\mathrm{~s})$, 134.3 (s), 169.2 (s), 169.1 (s), 168.4 (s), 165.6 (s), 119.6 (s), 109.8 (d), 139.0 (s), 166.0 (s), 66.1 (d), 76.5 (d), 69.2 (d), $73.6(d), 70.5(d), 64.3(q)$.

castalagin

(9). Off-white amorphous powder. m.p.243-245 ${ }^{\circ}$. FABMS m/z: $935[\mathrm{M}+\mathrm{H}]^{+}$and $933[\mathrm{M}-\mathrm{H}]^{-} \cdot{ }^{1} \mathrm{H}-\mathrm{NMR}\left(\left(\mathrm{CD}_{3}\right)\right.$ $\left.{ }_{2} \mathrm{CO}+\mathrm{D}_{2} \mathrm{O}\right) \delta: 6.66(1 \mathrm{H}, \mathrm{s}), 6.65(1 \mathrm{H}, \mathrm{s}), 6.52(1 \mathrm{H}, \mathrm{s}), 5.57$ $(1 \mathrm{H}, \mathrm{d}, \mathrm{J}=4.4 \mathrm{~Hz}), 4.97(1 \mathrm{H}, \mathrm{m}), 4.92(1 \mathrm{H}, \mathrm{m}), 5.04(1 \mathrm{H}, \mathrm{t}$, $\mathrm{J}=7.2 \mathrm{~Hz}), 5.44(1 \mathrm{H}, \mathrm{d}, \mathrm{J}=7.6 \mathrm{~Hz}), 3.94(1 \mathrm{H}, \mathrm{d}, \mathrm{J}=12.8)$, $4.80(1 \mathrm{H}, \mathrm{m}) .{ }^{13} \mathrm{C}-\mathrm{NMR}\left(\left(\mathrm{CD}_{3}\right)_{2} \mathrm{CO}+\mathrm{D}_{2} \mathrm{O}\right) \delta: 121.3(\mathrm{~s})$, 124.4 (s), 124.4 (s), 125.8 (s), 126.9 (s), 107.2 (d), 107.9 (d), 108.4 (d), 115.7 (s), 115.5 (s), 146.1 (s), 144.9 (s), 144.7 (s), 144.6 (s), 144.2 (s), 144.0 (s), 143.9 (s), 143.8 (s), 143.6 (s), 143.1 (s), 137.5 (s), 136.2 (s), 136.0 (s), $135.4(\mathrm{~s})$, 134.5 (s), 112.4 (s), 113.9 (s), 113.9 (s), 114.5 (s), 115.4 (s), 168.9 (s), 166.9 (s), 166.2 (s), 165.1 (s), 164.7 (s), 66.0 (d), $73.8(d), 66.6(d), 68.9(d), 70.9(d), 65.2(q)$.

\section{Discussion}

Flavonoids 1-5 and compound kaempferol-3-O- $\beta$-Dglucopyranoside were reported to have hypolipidemic, anti-oxidant, anti-inflammatory, analgesic, and antiaging activities [18]. The relationship between their structures and the efficiency was discussed. The presence of ortho-hydroxy group at B-ring, the double bond at $\mathrm{C} 2-\mathrm{C} 3$ for $\mathrm{C}$-ring, and the presence of $\mathrm{C}_{7}-\mathrm{OH}$ are usually listed as important conditions for high $\mathrm{AR}$ inhibitory effects. Accordingly, the double bond of C2-C3, ortho- or meta-hydroxy groups in B ring, or a glucose unit are associated with the enhanced cytotoxicity [19]. According to the data for compounds 1-6, the presence of $p$-coumaroyl groups would enhance the inhibitory activity in three assays, therein, flavonoids with more $p$ coumaroyl groups in structure are deemed to be the 
Table $3{ }^{1} \mathrm{H}-\mathrm{NMR}$ and ${ }^{13} \mathrm{C}-\mathrm{NMR}$ data of compounds 2-5

\begin{tabular}{|c|c|c|c|c|c|c|c|c|}
\hline \multirow[t]{2}{*}{ No. } & \multicolumn{2}{|l|}{2} & \multicolumn{2}{|l|}{3} & \multicolumn{2}{|l|}{4} & \multicolumn{2}{|l|}{5} \\
\hline & ${ }^{1} \mathrm{H}^{-N M R}{ }^{1}$ & ${ }^{13}$ C-NMR ${ }^{1}$ & ${ }^{1} \mathrm{H}^{-N M R}{ }^{1}$ & ${ }^{13}$ C-NMR $^{1}$ & ${ }^{1} \mathrm{H}-\mathrm{NMR}^{2}$ & ${ }^{13} \mathrm{C}-\mathrm{NMR}^{2}$ & ${ }^{1} \mathrm{H}-\mathrm{NMR}^{2}$ & ${ }^{13} \mathrm{C}-\mathrm{NMR}^{2}$ \\
\hline 2 & & 156.4 & & 156.4 & & 158.1 & & 158.7 \\
\hline 3 & & 133.0 & & 133.0 & & 134.6 & & 134.2 \\
\hline 4 & & 177.4 & & 177.4 & & 178.8 & & 178.8 \\
\hline 5 & & 156.3 & & 156.3 & & 162.8 & & 162.7 \\
\hline 6 & $6.15(1 \mathrm{H}, \mathrm{d}, 1.9)$ & 98.7 & $6.15(1 \mathrm{H}, \mathrm{d}, 1.9)$ & 98.7 & $6.05(1 \mathrm{H}, \mathrm{br} . \mathrm{s})$ & 100.4 & $6.05(1 \mathrm{H}, \mathrm{br} . \mathrm{s})$ & 99.7 \\
\hline 7 & & 164.1 & & 164.1 & & 165.4 & & 165.3 \\
\hline 8 & $6.39(1 \mathrm{H}, \mathrm{d}, 1.9)$ & 93.5 & $6.39(1 \mathrm{H}, \mathrm{d}, 1.9)$ & 93.5 & $6.24(1 \mathrm{H}$, br.s $)$ & 94.5 & $6.25(1 \mathrm{H}, \mathrm{br} . \mathrm{s})$ & 94.6 \\
\hline 9 & & 161.1 & & 161.1 & & 158.0 & & 158.0 \\
\hline 10 & & 103.9 & & 103.9 & & 105.7 & & 105.6 \\
\hline $1^{\prime}$ & & 120.7 & & 120.7 & & 122.6 & & 122.7 \\
\hline $2^{\prime}, 6^{\prime}$ & $7.99(2 \mathrm{H}, \mathrm{d}, 9.2)$ & 130.8 & $7.99(2 \mathrm{H}, \mathrm{d}, 9.2)$ & 130.8 & $7.89(2 \mathrm{H}, \mathrm{d}, 8.8)$ & 132.0 & $7.95(2 \mathrm{H}, \mathrm{d}, 8.8)$ & 131.9 \\
\hline $3^{\prime}, 5^{\prime}$ & $6.86(2 \mathrm{H}, \mathrm{d}, 9.2)$ & 115.1 & $6.86(2 \mathrm{H}, \mathrm{d}, 9.2)$ & 115.1 & $6.78(2 \mathrm{H}, \mathrm{d}, 8.8)$ & 116.0 & $6.86(2 \mathrm{H}, \mathrm{d}, 8.8)$ & 115.9 \\
\hline $4^{\prime}$ & & 160.0 & & 160.0 & & 161.2 & & 160.9 \\
\hline $1^{\prime \prime}$ & $5.45(1 \mathrm{H}, \mathrm{d}, 7.7)$ & 100.9 & $5.45(1 \mathrm{H}, \mathrm{d}, 7.7)$ & 100.9 & $5.57(1 \mathrm{H}, \mathrm{d}, 8.0)$ & 99.6 & $5.65(1 \mathrm{H}, \mathrm{d}, 8.4)$ & 100.3 \\
\hline $2^{\prime \prime}$ & $3.28(1 \mathrm{H}, \mathrm{d}, 7.7)$ & 74.2 & $3.28(1 \mathrm{H}, \mathrm{d}, 7.7)$ & 74.2 & $4.92(1 \mathrm{H}, \mathrm{d}, 8.0)$ & 75.7 & $5.09(1 \mathrm{H}, \mathrm{dd}, 8.8,8.4)$ & 75.6 \\
\hline $3^{\prime \prime}$ & $3.25(1 \mathrm{H}, \mathrm{d}, 7.7)$ & 76.2 & $3.25(1 \mathrm{H}, \mathrm{d}, 7.7)$ & 76.2 & $3.48 \mathrm{~m}$ & 76.2 & $3.71(1 \mathrm{H}, \mathrm{dd}, 8.8,8.8)$ & 76.1 \\
\hline $4^{\prime \prime}$ & $3.18(1 \mathrm{H}, \mathrm{m})$ & 69.9 & $3.18(1 \mathrm{H}, \mathrm{m})$ & 69.9 & $3.30 \mathrm{~m}(1 \mathrm{H}, \mathrm{d}, 8.8)$ & 71.5 & $3.44(1 \mathrm{H}, \mathrm{dd}, 8.8,8.8)$ & 71.9 \\
\hline $5^{\prime \prime}$ & $3.38(1 \mathrm{H}, \mathrm{m})$ & 74.1 & $3.38(1 \mathrm{H}, \mathrm{m})$ & 74.1 & $3.21 \mathrm{~m}$ & 78.6 & $3.59(1 \mathrm{H}, \mathrm{m})$ & 75.8 \\
\hline $6^{\prime \prime}$ & $4.28(1 \mathrm{H}, \mathrm{dd}, 12.0,2.0)$ & 63.0 & $4.28(1 \mathrm{H}, \mathrm{dd}, 12.0,2.0)$ & 63.0 & $3.67 \mathrm{~m}$ & 62.5 & $4.23(1 \mathrm{H}$, br.d, 10.8) & 64.1 \\
\hline & $4.03(1 \mathrm{H}, \mathrm{dd}, 12.0,2.0)$ & & $4.03(1 \mathrm{H}, \mathrm{dd}, 12.0,2.0)$ & & $3.46 \mathrm{~m}$ & & $4.37(1 \mathrm{H}, \mathrm{br} . \mathrm{d}, 10.8)$ & \\
\hline $1^{\prime \prime \prime}$ & & 124.9 & & 124.9 & & 127.1 & & 126.9 \\
\hline $2^{\prime \prime \prime}, 6^{\prime \prime \prime}$ & $7.37(2 \mathrm{H}, \mathrm{d}, 8.5)$ & 130.1 & $7.37(2 \mathrm{H}, \mathrm{d}, 8.5)$ & 130.1 & $7.34(2 \mathrm{H}, \mathrm{d}, 8.4)$ & 131.0 & $7.30(2 \mathrm{H}, \mathrm{d}, 8.8)$ & 131.0 \\
\hline $3^{\prime \prime \prime}, 5^{\prime \prime \prime}$ & $6.79(2 \mathrm{H}, \mathrm{d}, 8.5)$ & 115.6 & $6.79(2 \mathrm{H}, \mathrm{d}, 8.5)$ & 115.6 & $6.70(2 \mathrm{H}, \mathrm{d}, 8.4)$ & 116.6 & $6.80(2 \mathrm{H}, \mathrm{d}, 8.8)$ & 116.6 \\
\hline $4^{\prime \prime \prime}$ & & 159.8 & & 159.8 & & 160.9 & & 160.8 \\
\hline $7^{\prime \prime \prime}$ & $7.34(1 \mathrm{H}, \mathrm{d}, 15.8)$ & 144.6 & $7.34(1 \mathrm{H}, \mathrm{d}, 15.8)$ & 144.6 & $7.55(1 \mathrm{H}, \mathrm{d}, 16.0)$ & 146.6 & $7.40(1 \mathrm{H}, \mathrm{d}, 15.6)$ & 146.3 \\
\hline $8^{\prime \prime \prime}$ & $6.11(1 \mathrm{H}, \mathrm{d}, 15.8)$ & 113.6 & $6.11(1 \mathrm{H}, \mathrm{d}, 15.8)$ & 113.6 & $6.25(1 \mathrm{H}, \mathrm{d}, 16.0)$ & 115.0 & $6.07(1 \mathrm{H}, \mathrm{d}, 15.6)$ & 114.5 \\
\hline $9^{\prime \prime \prime}$ & & 166.1 & & 166.1 & & 168.1 & & 168.5 \\
\hline $1^{\prime \prime \prime \prime}$ & & & & & & & & 126.9 \\
\hline $2^{\prime \prime \prime \prime}, 6^{\prime \prime \prime \prime}$ & & & & & & & $7.48(2 \mathrm{H}, \mathrm{d}, 8.8)$ & 131.0 \\
\hline $3^{\prime \prime \prime \prime}, 5^{\prime \prime \prime \prime}$ & & & & & & & $6.80(2 \mathrm{H}, \mathrm{d}, 8.8)$ & 116.6 \\
\hline $4^{\prime \prime \prime \prime}$ & & & & & & & & 160.9 \\
\hline $7^{\prime \prime \prime \prime}$ & & & & & & & $7.72(1 \mathrm{H}, \mathrm{d}, 16.0)$ & 146.8 \\
\hline $8^{\prime \prime \prime \prime}$ & & & & & & & $6.44(1 \mathrm{H}, \mathrm{d}, 16.0)$ & 115.1 \\
\hline $9^{\prime \prime \prime \prime}$ & & & & & & & & 168.2 \\
\hline
\end{tabular}

promising anti-diabetes complications and anti-cancer agents.

Compounds 7, 8 and 9, as polyphenolic derivatives showed remarkable inhibitory effects in three bioactive systems, and the increasing number of galloyl groups in the structure could increase their activity. Some studies found that polyphenolic compounds possessed the potent anti-oxidant and anti-cancer activities to a greater or lesser extent [20], moreover, structure-activity relationships for their radical scavenging, anti-oxidant, anti-herbivore, and anti-herpetic activities also had been discussed [21-23]. Here, they were found having a healthy value against cancer or diabetic complications.

Based on our results, the anti-diabetes complications and anti-cancer activity of different parts of C. mollissina could be attributed to its containing amount of flavonoids and polyphenolic derivatives especially with more function groups in the structures. It is no doubt that as 
a food product the value for Ban li is not only for its nutrients but also for its functional values, including the value of its shells and involucres, which would be potential sources of phenolic compounds for using either as food additives or chemical medicines. We believe that systematic and thorough investigations on functions of Chinese chestnut are very necessary in future studies.

\section{Conclusions}

This paper aims to detail some standard procedures to provide better scope for performing the anti-diabetes complications and anti-cancer properties of C. mollissina. The plant is thus a promising source of anti-diabetes complications and anti-cancer drug besides indication that flavonoids and polyphenolic acids are the compounds responsible for these effects. Such findings are of extreme importance in the strive for future development of potent, safer and effective anti-diabetes complications and anticancer agent.

\section{Additional files}

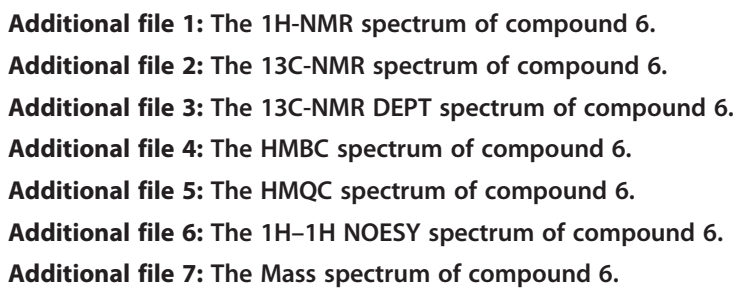

\section{Abbreviations}

CK: Alcohol extracts of kernels; CS: Alcohol extracts of shells; Cl: Alcohol extracts of involucres; CK-A: EtOAC soluble fractions of CK; CS-A: EtOAC soluble fractions of CS; Cl-A: EtOAC soluble fractions of $\mathrm{Cl} ; \mathrm{Cl}-\mathrm{B}$ : The $\mathrm{n}-\mathrm{BuOH}$ soluble fraction of $\mathrm{Cl}$; CK-B: The $\mathrm{n}-\mathrm{BuOH}$ soluble fractions of $\mathrm{CK}$; CS-B: The $\mathrm{n}-\mathrm{BuOH}$ soluble fractions of CS; CK-W: The aqueous parts of CK; CS-W: The aqueous parts of CS; CI-W: The aqueous parts of Cl; HR-AR: Human recombinant aldose reductase; AGEs: Advanced glycation end products; FBS: Fetal bovine serum; SMPC: Gly steroyl myristoyl phosphatidylcholine glycine; MTT: 3-(4, 5-dimethylthazol-2-yl)-2, 5-diphenyl tetrazolium bromidetetrazolium salt.

\section{Competing interests}

The authors declare that they have no competing interest.

\section{Author information}

LZ (Lin Zhang) and LZ (Libin Zhan) are associate professor and professor at Dalian medical University, respectively, Academy of Integrative Medicine and the Second Affiliated Hospital. HG and LW are associate professor and professor at Shenyang pharmaceutical University, respectively, Key Laboratory of Structure-Based Drug Design \& Discovery of Ministry of Education. MB, YO and TO are associate professor, professor and professor at Meiji Pharmaceutical University, respectively, Department of Natural Medicine and Phytochemistry.

\section{Authors' contributions}

$\mathrm{LZ}$ has performed the study and wrote the paper; TO, LW and YO has participated in the design of the study. LZ, MB and HG worked on the methods, data analysis and revised it critically. All authors read and approved the final manuscript.

\section{Acknowledgements}

We gratefully acknowledge the financial support by the project from Hebei Province Science Technology Program (2005), Meiji Pharmaceutical University Onda Scholarship for support of an international exchange program in pharmaceutical science and by the Program for Liaoning Innovative Research Team in University (no. LT2014018).

\section{Author details}

${ }^{1}$ Academy of Integrative Medicine, Dalian Medical University, No. 9, Western Section of South Road in Lvshun, Dalian 116044, China. ${ }^{2}$ Key Laboratory of Structure-Based Drug Design \& Discovery of Ministry of Education, Shenyang Pharmaceutical University, No.103, Wenhua Road, Shenhe District, Shenyang 110016, China. ${ }^{3}$ Department of Natural Medicine and Phytochemistry, Meiji Pharmaceutical University, No. 2-522-1, Noshio, Kiyose, Tokyo 204-8588, Japan. ${ }^{4}$ Department of Traditional Chinese Medicine, The Second Affiliated Hospital, Dalian Medical University, Dalian 116023, China.

Received: 2 June 2014 Accepted: 10 October 2014

Published: 28 October 2014

\section{References}

1. Zhang $J \mathrm{H}, \mathrm{He} Y \mathrm{~J}$ : Advances and development trends of Chinese chestnut research at home and abroad. World For Res 1999, 12:7-12.

2. Barreira JCM, Ferreira ICFR, Oliveira MBPP, Pereira JA: Antioxidant activities of the extracts from chestnut flower, leaf, skins and fruit. Food Chem 2008, 107:1106-1113.

3. Zhang DS, Gao HY, Wang LB, Li D, Kuroyanagi M, Wu LJ: Flavonol glycosides from Castanea Mollissima Blume. Asian J Tradit Med 2007, 25:229-234.

4. Long ZM, Wu LJ, Sun BH, Huang J, Gao HY: Chemical constituents from kernel of Castanea mollissima. J Shenyang Pharm Univ 2009, 26:614-616.

5. Long ZM, Wu LJ, Sun BH, Huang J, Gao HY: Chemical constituents from the seeds of Castanea mollissima Blume(III). J Shenyang Pharm Univ 2008, 25:883-885. 891 .

6. Lu C, Wu D, Gao HY, Sun BH, Huang J, Wu LJ: Isolation and identification of chemical constituents from testa of Castanea mollissima Blume. J Shenyang Pharm Univ 2010, 27:440-442. 462.

7. Amos AF, Mccarty DJ, Zimmet P: The rising global burden of diabetes and its complications: Estimates and projections to the year 2010. Diabetic Med 1997, 14:81-85.

8. Jemal A, Siegel R, Xu J, Ward E: Cancer statistics. CA Cancer J Clin 2010, 60:277-300.

9. Susanna CLM, Edward GM, Alicja WD: Diabetes and Colorectal Cancer Incidence in the Cohort of Swedish Men. Diabetes Care 2005, 28:1805-1807.

10. Mujić A, Grdović N, Mujić I, Mihailović M, Živković J, Poznanović G, Vidaković $\mathrm{M}$ : Antioxidative effects of phenolic extracts from chestnut leaves, catkins and spiny burs in streptozotocin-treated rat pancreatic $\beta$-cells. Food Chem 2011, 125:841-849.

11. Wu H, Dushenkov S, Ho CT, Sang SM: Novel acetylated flavonoid glycosides from the leaves of Allium ursinum. Food Chem 2009, 115:592-595.

12. Tian F, Li B, Jia BP, Zhang GZ, Luo YC: Identification and structure-activity relationship of gallotannins separated from Galla chinensis. Food Sci Tech 2009, 42:1289-1295.

13. Puttaiah S, Zhang YM, Pilch HA, Pfahler C, Oyaito T, Sayre LM, Nagaraj RH: Detection of dideoxyosone intermediates of glycation using a monoclonal antibody: characterization of major epitope structures. Arch Biochem Biophys 2006, 446:186.

14. Makita Z, Vlassara H, Ceramai HA, Bucala R: Immunochemical detection of advanced glycosylation end products in vivo. J Biol Chem 1992, 267:5133.

15. Cheng G, Bai YJ, Zhao YY: Flavonoids from Ziziphus jujuba Mill var. spinosa. Tetrahedron 2000, 56:8915-8920.

16. Ito $H$, Iguchi $A$, Hatano $T$ : Identification of urinary and intestinal bacterial metabolites of ellagitannin geraniin in rats. J Agric Food Chem 2008, 56:11668-11674.

17. Glabasnia A, Hofmann T: Sensory-directed identification of taste-active ellagitannins in American (Quercus alba L.) and European oak wood (Quercus robur L.) and quantitative analysis in bourbon whiskey and oak-matured red wines. J Agric Food Chem 2006, 54:3380-3390.

18. Huang AL: Studies on the activities of pharmacological effect of flavonoids. Anhui Agric Sci Bull 2007, 13:71-72 
19. Matsuda H, Morikawa T, Toguchida I, Yoshikawa M: Structural requirements of flavonoids and related compounds for aldose reductase inhibitory activity. Chem Pharm Bull 2002, 50:788-795.

20. Kunyanga CN, Imungi JK, Okoth M, Momanyi C, Biesalski HK, Vadivel V: Antioxidant and antidiabetic properties of condensed tannins in acetonic extract of selected raw and processed indigenous food ingredients from kenya. J Food Sci 2011, 76:560-567.

21. Cai YZ, Sun M, Xing J, Corke H: Antioxidant phenolic constituents in roots of rheum officinale and rubia cordifolia: structure-radical scavenging activity relationships. J Agric Food Chem 2004, 52:7884-7890.

22. Tian F, Li B, Jia BP, Zhang GZ, Luo YC: Identification and structure-activity relationship of gallotannins separated from Galla chinensis. Food Sci Tech 2009, 42:1289-1295.

23. Birt DF, Hendrich $S$, Wang W: Dietary agents in cancer prevention: Flavonoids and isoflavonoids. Pharmacol Ther 2001, 90:157-177.

doi:10.1186/1472-6882-14-422

Cite this article as: Zhang et al.: Extracts and compounds with antidiabetic complications and anti-cancer activity from Castanea mollissina Blume (Chinese chestnut). BMC Complementary and Alternative Medicine 2014 14:422.

\section{Submit your next manuscript to BioMed Central and take full advantage of:}

- Convenient online submission

- Thorough peer review

- No space constraints or color figure charges

- Immediate publication on acceptance

- Inclusion in PubMed, CAS, Scopus and Google Scholar

- Research which is freely available for redistribution 